\title{
The influence of glutamatergic receptor antagonists on biochemical and ultrastructural changes in myelin membranes of rats subjected to experimental autoimmune encephalomyelitis
}

\author{
Beata Dąbrowska-Bouta ${ }^{1}$, Lidia Strużyńska ${ }^{1}$, Małgorzata Chalimoniuk², Małgorzata Frontczak-Baniewicz ${ }^{3}$, \\ Grzegorz Sulkowski ${ }^{1}$ \\ ${ }^{1}$ Laboratory of Pathoneurochemistry, Department of Neurochemistry, ${ }^{2}$ Department of Cellular Signaling, ${ }^{3}$ Electron Microscopy \\ Platform, Mossakowski Medical Research Centre Polish Academy of Sciences, Warsaw, Poland
}

\begin{abstract}
Elevated extracellular glutamate in the synaptic cleft causes overactivation of glutamate receptors and kills neurons by an excitotoxic mechanism. Recent studies have shown that glutamate can also lead to toxic injury of white matter oligodendrocytes in myelin sheaths and consequently to axon demyelination. The present study was performed using the rodent model of multiple sclerosis known as experimental autoimmune encephalomyelitis (EAE). The aim of the study was to test the effects of the glutamatergic receptor antagonists amantadine and memantine (antagonists of NMDA receptors), LY 367384 (an antagonist of MGluR1), and MPEP (an mGluR5 antagonist) on the development of neurological symptoms in immunized animals, morphological changes in cerebral myelin, and expression of $m R N A$ of the principal myelin proteins PLP, MBP, MOG, MAG, and CNPase. Pharmacological inhibition of NMDA receptors by amantadine and memantine was found to suppress neurological symptoms in EAE rats, whereas antagonists of the group I metabotropic glutamate receptors ( $m G l u R s ~ G I$ ) did not function positively. In the symptomatic phase of the disease we observed destruction of myelin sheaths via electron microscopy and decreased levels of mRNA for all of the principal myelin proteins. The results reveal that glutamate receptor antagonists have a positive effect on the expression of mRNA MBP and glycoproteins MAG and MOG but not on myelin ultrastructure.
\end{abstract}

Key words: EAE, glutamate receptors antagonists, myelin proteins, excitotoxicity.

\section{Introduction}

Glutamate is the primary excitatory amino acid neurotransmitter in the mammalian brain. It plays an important role in both physiological and pathological mechanisms operating in the central nervous system (CNS). Glutamate released from nerve end- ings participates in signaling processes prior to being taken up from the synaptic cleft via a mechanism mediated mainly by glial transporters and to a lesser extent neuronal, excitatory amino acid transporters (EAATs) $[10,27]$. The extracellular levels of glutamate must be tightly controlled because excessive accumulation of glutamate leads to overstimulation of

Communicating author:

Grzegorz Sulkowski, Laboratory of Pathoneurochemistry, Department of Neurochemistry, Mossakowski Medical Research Centre Polish Academy of Sciences, 5 Pawińskiego St., 02-106 Warsaw, Poland, phone: +48 2260865 30, fax: + 48226085423 ,

e-mail: gsulkowski@imdik.pan.pl 
glutamate receptors (GluRs) and subsequent cell damage by an excitotoxic mechanism [6,8,29].

Excitotoxicity is implicated in the pathomechanism of a number of chronic neurodegenerative diseases such as Alzheimer's disease, Parkinson's disease, Huntington's disease, and amyotrophic lateral sclerosis (ALS) $[2,7,8,28]$. Although the pathology of each of these diseases is different, each involves overstimulation of GluRs (especially the N-methyl-D-aspartate receptor [NMDAR] subtype). Such overstimulation ultimately leads to injury of neurons by necrotic or apoptotic cell death [13,32]. The pathomechanism of excitotoxicity also operates in multiple sclerosis (MS), wherein the immune system attacks the CNS. MS is characterized by infiltration of immune cells from peripheral circulation, loss of oligodendrocytes and the appearance of demyelinating areas (plaques) in the white matter of the spinal cord and brain. These disorders lead to axonal damage and disrupted neurotransmission [13,17,23]. The etiology of MS has not yet been established. Many pathological factors participate in the pathogenesis of MS, including proinflammatory cytokines, reactive oxygen species (ROS), matrix metalloproteinases, autoantibodies and cell-mediated cytotoxicity $[13,19]$.

Enhancement of glutamate levels in cerebrospinal fluid and changes in the expression of metabotropic (mGluRs) and ionotropic (iGluRs) glutamate receptors have been observed in the inflammatory demyelination plaques in the brains of MS patients $[12,13]$. Elevation of glutamate in brain was also observed in experimental autoimmune encephalomyelitis (EAE, the animal model of MS) pathology in rats [6]. The hypothesis concerning the involvement of excitotoxicity in pathogenesis of MS and EAE has been confirmed by the observation that anti-glutamatergic agents provide neuroprotection [28]. Our previous studies using EAE rats revealed that administration of amantadine or memantine (iGluR antagonists) reduces the severity of symptoms, inflammation and axonal damage in immunized animals [33-35]. This is a strong indication that glutamate, operating through its ionotropic receptors, plays a key role in the pathology of the disease. If we can gain an understanding of how myelin and oligodendrocytes are damaged by excitotoxicity, we will be able to develop therapeutic strategies to protect nerve axons against demyelination.

The myelin sheath is the predominant element of the white matter of the CNS, its function being to facilitate signal conduction in axons. Myelin is a highly specialized structure with a unique molecular composition and architecture. It is characterized by a high proportion of lipids (70-85\%) and a low proportion of proteins (15-30\%) [25]. It contains distinctive proteins such as proteolipid protein (PLP), myelin basic protein (MBP), 2',3'-cyclic nucleotide 3'-phosphodiesterase (CNPase), myelin-associated glycoprotein (MAG) and myelin oligodendrocyte glycoprotein (MOG). PLP and MBP constitute the majority of the total myelin proteins (about 70\%) [4,25]. CNPase is a specific enzyme localized in the cytoplasm of non-compacted myelin. MOG is a transmembrane protein representing one of the main autoantigens in MS. MAG is a glycoprotein involved in the myelin-related inhibition of axonal regeneration. Each of these myelin-specific proteins plays an important role in the formation and maintenance of myelin membranes [4,5,31].

The present study was undertaken to investigate whether the glutamate receptor antagonists amantadine and memantine (antagonists of NMDA receptors), LY 367384 (an antagonist of mGluR1) and MPEP (an mGluR5 antagonist) improve the condition of animals subjected to EAE and exert protective effects on mRNA expression of selected myelin proteins (PLP, MBP, MOG, MAG, and CNPase). Ultrastructural observations of myelin membranes in the acute phase of EAE and after treatment with GluR antagonists were also made using transmission electron microscopy (TEM).

\section{Material and methods \\ Animal model}

The study was carried out in strict accordance with the regulations of the Experiments on Animals Act (Act of 21 January 2005 on experiments on live animals, the Parliament of the Republic of Poland, Dz. U. Nr 33, pos. 289). All experiments using animals were approved by the Fourth Warsaw Local Ethics Committee for Animal Experimentation; permit number $61 / 2009$. All procedures using animals were performed under sodium pentobarbital anesthesia to minimize suffering.

Female Lewis rats weighing approximately $200 \mathrm{~g}$ were used. To induce EAE, we immunized rats subcutaneously in both hind feet with $100 \mu \mathrm{l}$ of inoculum containing guinea pig spinal cord homogenate emulsified in Freund's complete adjuvant (CFA) containing 
$5.5 \mathrm{mg} / \mathrm{ml}$ Mycobacterium tuberculosis H37Ra (Difco, Detroit, Mi, USA). The control group received inoculum containing CFA without spinal cord homogenate.

After immunization, the rats were provided with unrestricted access to food and water and were housed under environmentally controlled conditions. Body weight and neurological deficits were measured daily according to the following scale: $0=$ no signs, 1 = flaccid tail, 2 = impairment of fighting reflex and/ or loss of muscle tone in hind limbs, $3=$ complete paralysis of hind limbs, $4=$ paraplegia, and $5=$ moribund state/death $[15,26]$.

\section{Experimental groups and tissue processing}

All experiments were performed during the acute phase of the disease at day 12 post immunization (d.p.i.). Six experimental groups of animals were defined: group I (control), group II (EAE), group III (EAE + amantadine), group IV (EAE + memantine), group V (EAE + LY 367385), and group VI (EAE + MPEP). During the experiments the part of rats in each experimental group $(n=12)$ were monitored until day 25 after the initial injection inducing EAE or after drug administration.

Glutamate receptor antagonists were dissolved in PBS and administered via intraperitoneal injection to the EAE rats once daily for 7 consecutive days, from 5 d.p.i. to 11 d.p.i., according to the previously described procedure [33-35]. Amantadine (Sigma-Aldrich, Steinheim, Germany) was administered at a dose of $100 \mathrm{mg} / \mathrm{kg}$ b.w./day, memantine (Sigma-Aldrich, Steinheim, Germany) was administered at a dose of $60 \mathrm{mg} / \mathrm{kg}$ b.w./day, and LY 367385 (Tocris, Bristol, UK) and MPEP (Tocris, Bristol, UK) were both administered at a dose of $10 \mathrm{mg} / \mathrm{kg}$ b.w./day.

At 12 d.p.i. (in the acute, symptomatic phase of the disease, when the neurological deficits were maximal), eight rats were sacrificed in each experimental group for real-time PCR analysis. The brains were rapidly removed, frozen in liquid nitrogen and stored at $-70^{\circ} \mathrm{C}$ for further analysis (extraction of RNA).

\section{Determination of mRNA levels of myelin proteins by real time-PCR}

Total RNA was extracted from the brain cortex of all six groups of rats according to the method of Chomczyński [9] using TRI Reagent (Sigma, St. Louis, MO, USA). Reverse transcription of $2 \mu \mathrm{g}$ of total RNA was performed in a final volume of $20 \mu \mathrm{l}$ using random primers and avian myeloblastosis virus (AMV) reverse transcriptase (Life Technologies, Forest City, CA, USA). The RT-PCR conditions were as follows: reverse transcription at $42^{\circ} \mathrm{C}$ for $45 \mathrm{~min}$ and denaturation at $94^{\circ} \mathrm{C}$ for $30 \mathrm{~s}$. For quantitative real-time $\mathrm{PCR}$ analysis, TaqMan technology was applied. Rat myelin protein-specific primers for MOG Rn 00575354 m1, PLP Rn00456892_m1, MBP Rn01399619_m1, MAG Rn02586362, CNPase Rn01399463_m1 and the corresponding probes were obtained from Life Technologies (Forest City, CA, USA). The mRNA expression levels of myelin proteins and actin were determined using the pre-validated TaqMan assay reagents (Applied Biosystems, Forest City, CA, USA). Real-time PCR was conducted on an ABI Prism 7500 system using $5 \mu \mathrm{l}$ of RT product, TaqMan PCR Master Mix, primers, and a TaqMan probe in a total volume of $20 \mu \mathrm{l}$. The PCR cycle conditions were as follows: initial denaturation at $95^{\circ} \mathrm{C}$ for $10 \mathrm{~min}, 50$ cycles of $95^{\circ} \mathrm{C}$ for $15 \mathrm{~s}$, and $60^{\circ} \mathrm{C}$ for $1 \mathrm{~min}$. Each sample was analyzed in triplicate. The relative expression levels of the myelin protein mRNAs were calculated using a standard curve and normalized to actin.

\section{Electron microscopic studies (TEM)}

The analysis of ultrastructural changes in brain myelin was performed at 12 d.p.i. using five animals from each experimental group (control, EAE, EAE + amantadine, EAE + memantine, EAE + LY 368573, and $E A E+M P E P)$. The animals were anaesthetized and perfused through the heart with fixative solution ( $2 \%$ paraformaldehyde, $2.5 \%$ glutaraldehyde, and $0.1 \mathrm{M}$ cacodylate buffer, $\mathrm{pH}$ 7.4). After perfusion, small specimens from the forebrain were fixed overnight in the same solution and then post-fixed in $1.5 \% \mathrm{OsO}_{4}$ and $0.8 \% \mathrm{~K}_{4}(\mathrm{FeCN})_{6}$ for $2 \mathrm{~h}$. Then the material, after dehydration in ethanol and propylene oxide, was embedded in Spurr resin. Ultrathin sections were examined using a JEM 1200 Ex electron microscope.

\section{Statistical analysis}

The results are expressed as means \pm SD from 3-8 experiments as identified in the legends of respective figures. Statistical significance was assessed by one-way ANOVA. Dunnett's multiple comparison test was used to identify the changes deemed to be significantly different from the control values. 


\section{Results}

\section{The influence of glutamate receptor antagonists on the course of $E A E$}

The modulatory effects of glutamate receptor antagonists (amantadine, memantine, LY 367385, and MPEP) on the course of EAE, changes in body mass, duration of the disease, and inductive phase of EAE (characteristic parameters of the disease) were investigated. All studies were performed during the acute phase of EAE at 12 d.p.i., while the neurological deficits were maximal. We observed a 30\% loss of body mass in EAE rats compared to the control animals. In groups treated with amantadine or memantine the loss of body mass was lower. We observed about a $20 \%$ decrease relative to the con-
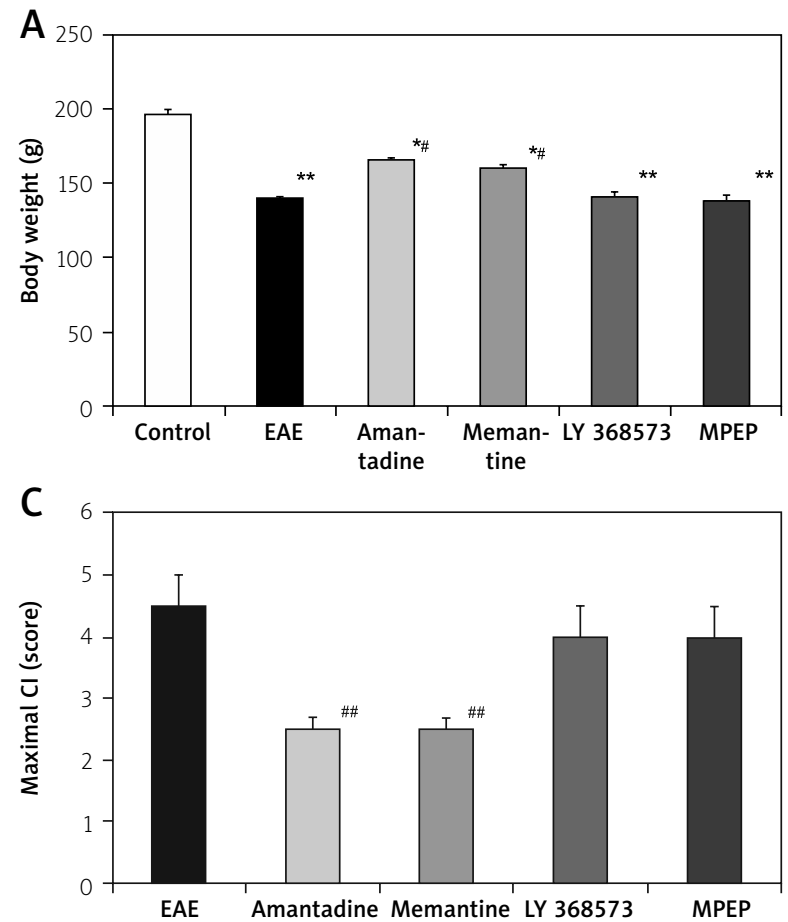

trol, and about a $10 \%$ increase compared to EAE rats in body weight (Fig. 1A). Neurological deficits during the course of EAE were classified daily according to a scale from $1+$ to $5+$ as described in the Materials and Methods section. After administration of either memantine or amantadine, we observed a reduction in duration of neurological deficits (Fig. 1B) and in the severity of the disease (lowering of the score) (Fig. 1C) compared with EAE rats. Paralysis of the tail and hind limbs, reduction of muscle tone and physical activity in experimental rats were exclusively observed. The maximal cumulative index ( $\max \mathrm{Cl})$ value reached $4.5+$ in EAE rats, whereas in the amantadine- and memantine-treated groups it was $2.5+$ (Fig. 1C). All rats in both of these groups exhibited improved physiological conditions relative
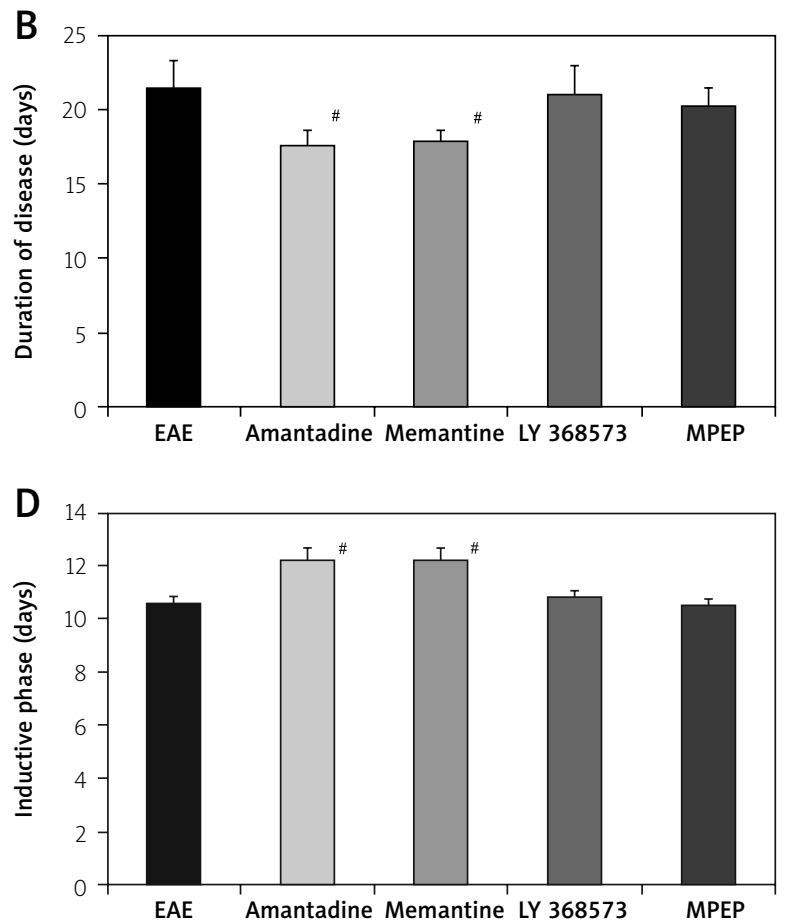

Fig. 1. Scores of the neurological symptoms and characteristic disease parameters observed in the acute phase of EAE (at 12 d.p.i.) and after treatment with antagonists of glutamate receptors. Neurological signs were monitored in the part of rats in each experimental group $(n=12)$ until recovery of the control EAE group (at 25 d.p.i.). Doses of antagonist were as follows: amantadine $100 \mathrm{mg} / \mathrm{kg}$ b.w./day, memantine $60 \mathrm{mg} / \mathrm{kg}$ b.w./day, LY $36738510 \mathrm{mg} / \mathrm{kg}$ b.w./day and MPEP $10 \mathrm{mg} / \mathrm{kg}$ b.w./day. The antagonists were administered once daily for 7 consecutive days, starting from 5 d.p.i. to 11 d.p.i. The graphs indicate: A) body weight of animals; B) duration of the disease; C) maximal cumulative index (score); D) duration of inductive phase of the disease. Results were obtained from five to eight animals in each group and represent means $\pm \mathrm{SD} .{ }^{*} p<0.05,{ }^{* *} p<0.01$ significantly different vs. control (healthy untreated rats). ${ }^{\#} p<0.05$ significantly different vs. EAE rats not subjected to therapy at 12 d.p.i. (one-way ANOVA followed by Dunnett's multiple comparison post test). 
to the untreated EAE animals. The duration of the inductive phase of the disease (Fig. 1D) was lengthened by 1-2 days, whereas the total disease duration (Fig. 1B) was reduced by 2-4 days, relative to the untreated EAE rats. We did not observe any neuroprotective effects of LY 367385 or MPEP with respect to body mass, neurological deficits or duration of the disease. In these groups, all examined parameters were found to be statistically insignificant compared with the untreated EAE rats (Fig. 1A-D).

Analysis of the clinical parameters of the disease, as well as the effects of GluR antagonist administration on neurological deficits and the condition of the EAE rats during longer experiments (until 25 d.p.i.), are described and illustrated in detail in our previous papers [33-35].

\section{Influence of drug administration on expression of mRNA of myelin proteins in $\mathrm{EAE}$ rats}

We investigated the mRNA levels of myelin-specific proteins: PLP and MBP (structural proteins); MOG and MAG (myelin glycoproteins); and the enzyme CNPase (a myelin marker). The analysis of mRNA was conducted in the forebrains of rats obtained from all experimental groups (control, $E A E$, and EAE treated with antagonists of glutamate receptors) by real-time PCR.

In the EAE rats, at the peak of the disease, we observed significantly lower levels of PLP and MBP mRNA, which reached about $70 \%$ and $60 \%$ of control values, respectively (Fig. 2A-B). In the groups treated with amantadine or memantine, the PLP mRNA level was found to be similar to that observed in the untreated EAE rats. Administration of LY 367385 (the mGluR1 antagonist) and MPEP (the mGluR5 antagonist) resulted in a further decrease of PLP mRNA, to $90 \%$ of the control value, and to $50 \%$ of the value observed in the untreated EAE rats (Fig. 2A). The level of MBP mRNA was about $40 \%$ lower than that of the control but about $40 \%$ higher than that of the EAE rats in all groups treated with GluR antagonists (amantadine, memantine, LY 367385, and MPEP) (Fig. 2B).

The analysis of expression of MOG and MAG mRNAs in EAE animals indicated a $60 \%$ and a $70 \%$ decrease, respectively, relative to the control value (Fig. 2C, 2D). In groups treated with amantadine or memantine, the mRNA level of MOG was found to be similar to that of the untreated EAE rats, but in the LY 367385- or MPEP-treated rats the MRNA level increased to about $50 \%$ of that of the untreated EAE animals (Fig. 2C). An increased level of MAG mRNA was also observed in all treated groups (Fig. 2D). After administration of amantadine or memantine the level of MAG mRNA was found to be $20 \%$ higher, whereas in LY 367385- and MPEP-treated groups the MAG mRNA level was found to decrease by about $50-60 \%$ of the MAG mRNA level of the untreated EAE rats (Fig. 2D). Our studies also revealed changes in the level of CNPase mRNA in all experimental groups compared with control animals. We observed a statistically significant decrease in the CNPase mRNA level, which reached $60-70 \%$ of the control value. Administration of all tested GluR antagonists resulted in a decrease in CNPase mRNA expression by $30 \%$ relative to the level of CNPase mRNA expression in untreated EAE rats (Fig. 2E).

\section{Electron microscopic analysis}

TEM analysis was performed in forebrain specimens obtained from rat brains during the acute phase of EAE at 12 d.p.i. In control rats, the myelin exhibited normal lamellar ultrastructure characterized by ordered layers closely adhering to each other (Fig. 3A). In large areas of untreated EAE rat brains (Fig. 3B), as in brains of rats from all experimental groups treated with the GluR antagonists amantadine (Fig. 3C), memantine (Fig. 3D), LY 367385 (Fig. 3E), and MPEP (Fig. 3F), we observed visible changes in the myelin membranes. The characteristic compact myelin structure was essentially completely destroyed. Multilayered membranes of myelin sheaths were irregular and loosely arranged. Administration of GluR antagonists did not provide protective effects on the ultrastructure of myelin relative to the untreated EAE rats (Fig. 3B-F).

\section{Discussion}

Recent studies have demonstrated that glutamate excitotoxicity is an important mechanism which is related to tissue injury in MS lesions [3,6,22,38]. Any imbalance in glutamate homeostasis in MS, either by increased production, reduced transport or impaired metabolism, is likely to affect oligodendrocytes [38] and cells producing myelin sheaths that support electrical conduction in the CNS [25].

The present study investigates whether administration of glutamate receptor antagonists to EAE 

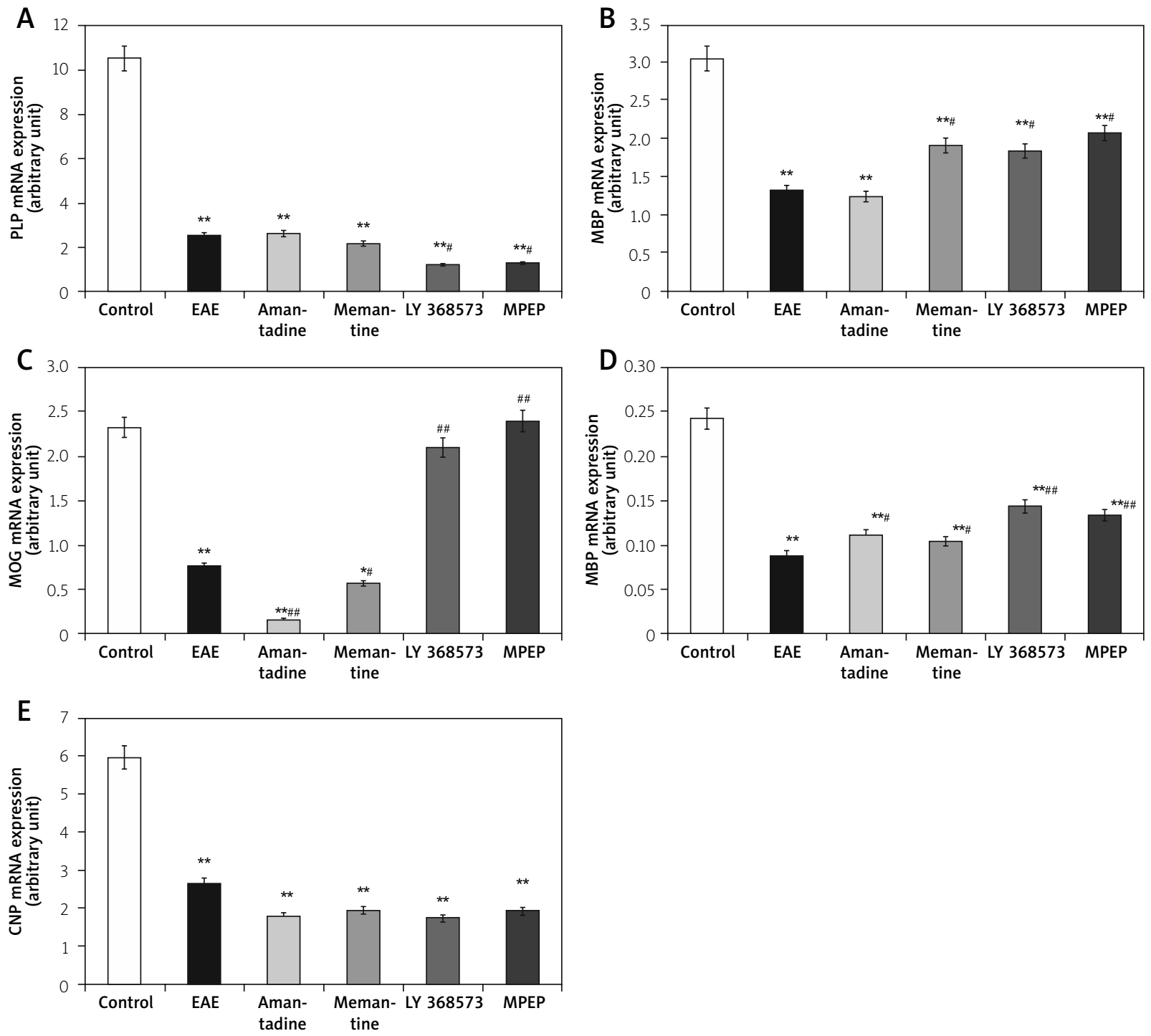

Fig. 2. Expression of mRNA of myelin proteins in the forebrains of control, EAE rats, and EAE rats after treatment with antagonists of glutamatergic receptors: amantadine, memantine, LY 367385, and MPEP) in the acute phase of the disease (at 12 d.p.i.). The mRNA levels of A) PLP, B) MBP, C) MOG, D) MAG, and E) CNPase were determined by quantitative real time-PCR (see Material and methods) and normalized against actin. Graphs indicate the results expressed as arbitrary units from four independent experiments, each performed using distinct brain samples. The values represent the means $\pm \mathrm{SD},{ }^{*} p<0.05,{ }^{* *} p<0.01$, significantly different vs. control rats. ${ }^{\#} p<0.05$, ${ }^{\# \#} p<0.01$, significantly different vs. EAE rats not subjected to therapy (one-way ANOVA followed by Dunnett's multiple comparison post-test).

rats may exert an effect on the development of neurological symptoms during EAE or improve the quality of myelin membranes at the biochemical level and the ultrastructural level. We tested two antagonists of NMDA receptors (amantadine and memantine), an antagonist of mGluR1 (LY 367384) and an antagonist of mGluR5 (MPEP) during the acute phase of EAE.
Reduction in the strength of neurological symptoms of EAE was observed only in animals treated with the NMDAR antagonists. Administration of either amantadine or memantine resulted in a reduction of both severity and duration of the neurological deficits. The duration of the inductive phase of the disease and the length of the disease were reduced by 2 and 4 days, respectively, relative 

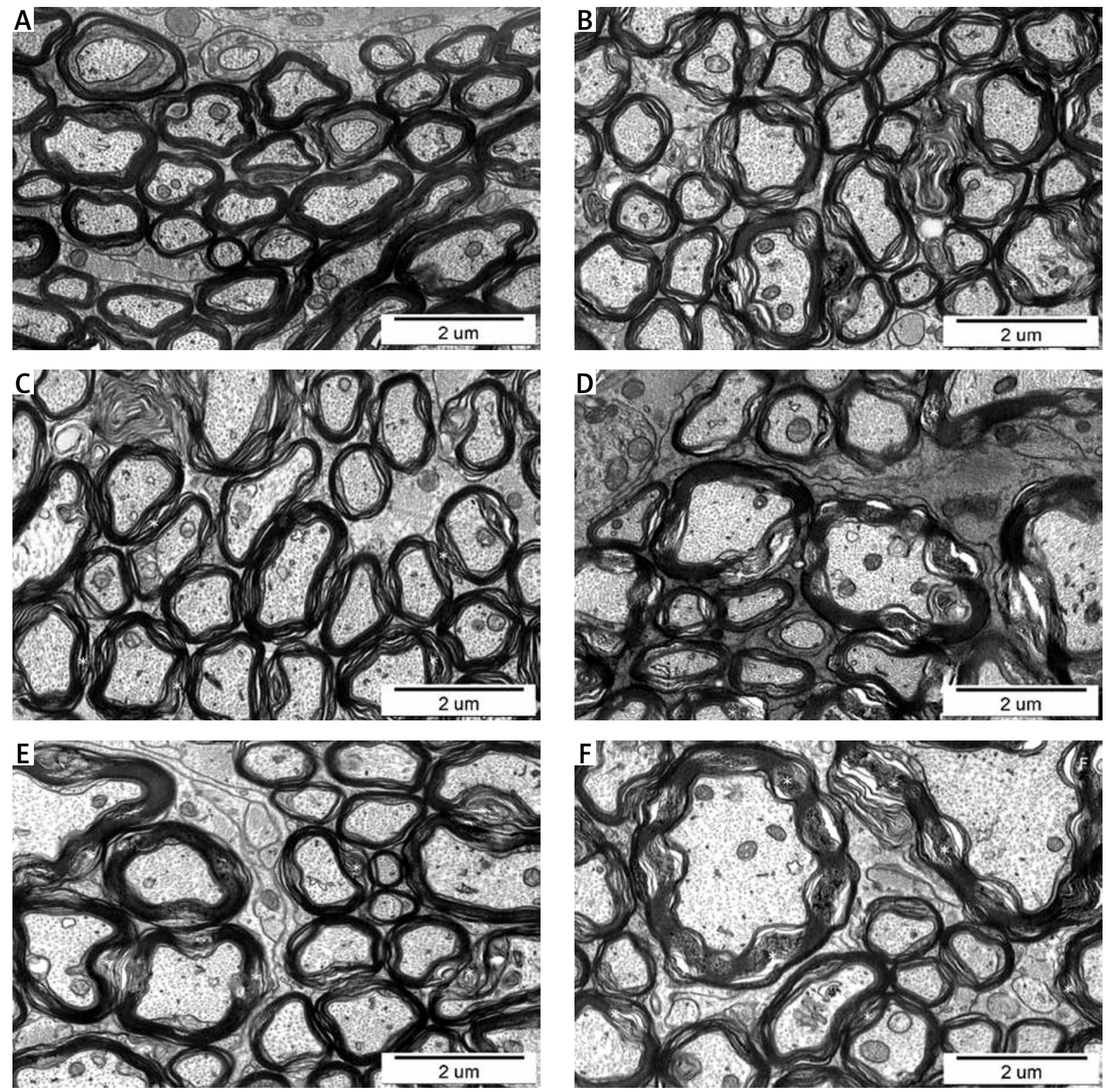

Fig. 3. Representative electron micrographs of myelin in forebrain specimens obtained from control and EAE rats untreated or treated with antagonists of the glutamatergic receptors amantadine, memantine, LY 367385, and MPEP) in the acute phase of EAE (at 12 d.p.i.). A) Control rats, B) EAE rats, C) EAE + amantadine, D) $E A E+$ memantine, E) EAE + LY 367385 and F) EAE + MPEP. Asterisks mark disintegrated lamellar structure of myelin membranes.

to the untreated EAE rats. Rats treated with amantadine and memantine also generally retained better physiological conditions relative to the untreated EAE animals. Administration of LY 367385 or MPEP did not result in any improvement of the neurological parameters of EAE animals.

Glutamatergic NMDA receptors play a vital role in maintaining normal synaptic transmission $[6,21]$.
Specific prevention of the pathological activation of NMDA receptors with drugs such as memantine or amantadine reduces undesirable activity and thereby improves the neurological conditions of EAE rats. Amantadine and memantine have been previously found to be effective in different models of excitotoxic damage in both in vivo and in vitro experiments $[1,28,36,37]$. 
It was also of interest to determine whether administration of GluR antagonists to EAE rats would simultaneously improve animals' condition and have a positive effect on the destroyed brain myelin. Myelin is a multilayered stack of thick membranes with alternating electron-dense and electron-light layers (the major dense line and the intraperiod line, respectively). These layers can be seen by electron microscopy [14]. We observed these layers in electron micrographs of control rat brain tissue (Fig. 3A). This membrane is formed as an extension of the plasma membrane of oligodendrocytes in the CNS and provides the insulation required to facilitate rapid signal transmission between neurons [4]. Numerous electron microscopy studies have demonstrated degeneration of myelin sheaths in MS and EAE models $[18,20,22]$. Our observations of disturbed myelin ultrastructure are consistent with observations in these previous studies. In contrast to our expectations, none of the applied glutamate receptor antagonists were found to exert a protective effect on the myelin ultrastructure. Characteristics of myelin damage were similar in all of the treatment groups. We also observed focal detachment of the myelin lamellae and increased volumes of cytoplasm in the regions of mature compacted myelin.

Subsequently we investigated whether the antagonists of glutamate receptors (NMDA and mGluRs) may exert an effect on biochemical parameters of myelin during the acute phase of EAE. We analyzed the expression of all primary proteins, which include proteolipid protein, myelin basic protein, 2',3'-cyclic nucleotide-3'-phosphohydrolase, myelinassociated glycoprotein, and myelin/oligodendrocyte glycoprotein. The results indicate that the expression of myelin proteins in all experimental groups is altered. The level of mRNA of each of the tested proteins was found to be reduced in EAE rats compared to the controls. However, treatment with the glutamate receptor antagonists was found to alter expression levels of the mRNAs. We observed an increase in the level of MBP mRNA in all of the therapeutic groups. These increases were in the range of $30 \%-50 \%$ relative to the untreated $\mathrm{EAE}$ rats. MBP is a major protein of CNS myelin, constituting as much as $30 \%$ of total protein and playing a pivotal role in myelin compaction. It also stabilizes the major dense line to facilitate the adhesion of the membrane layers [4,31]. Conversely, we did not observe increased levels of PLP mRNA after admin- istration of glutamate receptor antagonists. PLP is another major myelin constituent (about 50\% of myelin proteins) whose role is to maintain separation between the layers of myelin membranes. Possibly as a result of its key role in maintaining myelin structure, the improvement was not visible in TEM, although the expression of MBP was found to increase simultaneously. Furthermore, we analyzed expression of 2',3'-cyclic nucleotide-3'-phosphohydrolase (CNPase), an enzyme specifically expressed in myelin and located in oligodendrocytes, mainly around the nucleus and in the paranodal loops [31]. CNPase may have an additional enzymatic function unrelated to its hydrolase activity, for which no physiological substrate has been found. The protein exists in two splice variants and constitutes 3-4\% of total myelin protein. In the case of CNPase mRNA level we did not observe protective efficacy of tested GluR antagonists. Administration of both NMDARs and $\mathrm{mGluR}$ antagonists caused a decrease in CNPase mRNA expression by about $30 \%$ relative to EAE rats.

Interestingly, mRNA expression levels of the glycoproteins MAG and MOG were found to be altered. MAG is a large (approximately $100 \mathrm{kDa}$ ) protein and is quantitatively a minor constituent, representing only $1 \%$ of the total myelin protein. This protein is located at the inner surface of the myelin sheath opposing the axon surface [16]. MOG is a transmembrane glycoprotein which constitutes $0.01-0.05 \%$ of total myelin proteins [4] and is located on the outer surface of the membrane of oligodendrocytes. This protein is widely used to induce EAE [30]. We observed that glutamate receptor antagonists modify the expression of both of these myelin glycoproteins. After treatment with amantadine or memantine, MOG mRNA levels were found to be similar to the levels expressed in EAE rats, whereas LY 367385 and MPEP resulted in increased expression of MOG mRNA relative to EAE rats. The levels of MAG mRNA were found to increase in all groups of animals treated with GluR antagonists. Although oligodendrocytes possess both NMDAR and mGluR classes of glutamatergic receptors [11,24], exclusively group I (mGluR G I) was shown to play an important role in regulation of MOG mRNA expression.

The results of our experiments confirm the involvement of glutamate in the pathology of EAE. Not all neurological symptoms and biochemical changes in the expression of basic myelin proteins were abolished, but some of the tested parameters 
showed statistically significant improvements after administration of glutamate receptor antagonists. Our previous studies demonstrated that glutamate excitotoxicity is one of the pathomechanisms implicated in the acute phase of EAE. Treatment of EAE rats with antagonists of both group I mGluRs (LY 367385 and MPEP) and NMDARs (amantadine and memantine) was found to modulate the expression of mRNA and proteins for both types of glutamate receptors, to decrease the expression of proinflammatory cytokines, to modulate glutamate transport (uptake and release) in nerve endings, and to reduce the levels of mRNA of glutamate transporters (EAATs) $[34,35]$. The present study indicates that glutamate excitotoxicity is a crucial factor involved in the process of myelin destruction during EAE. This effect is a separate and distinct effect from neuroinflammation.

\section{Conclusions}

Antagonists of NMDARs exert a neuroprotective effect and inhibit neurological deficits in EAE rats. Both amantadine and memantine reduce the severity of neurological deficits, maximal score, and duration of disease. A neuroprotective effect was not observed after administration of group I mGluR antagonists (LY 367385 and MPEP). Neither the general condition of EAE rats nor the neurological deficits were improved.

Ultrastructural studies showed that in EAE rats the characteristic multilamellar structure of myelin is destroyed. We did not observe protective effects on the morphology of myelin membranes after administration of GluR antagonists. At the peak of the disease we observed a significant decrease in $\mathrm{mRNA}$ levels for each of the investigated myelin proteins (PLP, MBP, MOG, MAG, and CNPase) in EAE rats relative to the controls. The mRNA expression was found to be modified in EAE rats treated with the GluR antagonists. After treatment with each of the GluR antagonists, the expression levels of MBP mRNA and MAG mRNA were found to increase significantly, whereas the expression levels of CNPase mRNA were found to decrease relative to untreated EAE animals. After amantadine or memantine administration PLP mRNA and MAG mRNA expression levels were unchanged compared with untreated EAE rats. Administration of mGluR G I antagonists (LY 367385 and MPEP) was found to increase the mRNA expression levels of MAG glycoprotein, whereas the expression levels of PLP MRNA were found to decrease relative to the untreated EAE rats. Changes in mRNA expression of myelin proteins which are subsequently altered by administration of GluR antagonists may indicate participation of glutamate-mediated excitotoxicity in myelin degradation during the acute phase of EAE.

\section{Acknowledgments}

This study was partially supported by grant no. NN401 620038 from the Polish Ministry of Science and Higher Education.

\section{Disclosure}

Authors report no conflict of interest.

\section{References}

1. Abdurasulova IN, Serdyuk SE, Gmiro VE. Combined blockade of GLUR1 AMPA and NMDA receptors effectively eliminates neurological disorders in rats with experimental allergic encephalomyelitis. Eksperimentalnaya i Klinicheskaya Farmakologiya 2007; 70: 15-19.

2. Anneser JMH, Barasio GD, Berthele A, Zieglgansberger W, Tolle TR. Differential expression of group I metabotropic glutamate receptors in rat spinal cord somatic and autonomic motoneurons: possible implications for the pathogenesis of amyotrophic lateral sclerosis. Neurobiol Dis 1999; 6: 140-147.

3. Basso AS, Frenkel D, Quintana FJ, Costa-Pinto FA, Petrovic-Stojkovic S, Puckett L, Monsonego A, Bar-Shir A, Engel Y, Gozin M, Weiner HL. Reversal of axonal loss and disability in a mouse model of progressive multiple sclerosis. J Clin Invest 2008; 118: 1532-1543.

4. Baumann N, Pham-Dinh D. Biology of oligodendrocyte and myelin in the mammalian central nervous system. Physiol Rev 2001; 81: 871-927.

5. Berger T, Rubner P, Schautzer F, Egg R, Ulmer H, Mayringer I, Dilitz E, Deisenhammer F, Reindl M. Antimyelin antibodies as a predictor of clinically definite multiple sclerosis after a first demyelinating event. N Engl J Med 2003; 349: 139-145.

6. Bolton C, Paul C. Glutamate receptors in neuroinflammatory demyelinating disease. Mediators Inflamm 2006; 2: 93684.

7. Bradley SR, Marino MJ, Wittmann M, Rouse ST, Awad H, Levey $A E$, Conn PJ. Activation of group II metabotropic glutamate receptors inhibits synaptic excitation of the substantia nigra pars reticulate. J Neurosci 2000; 20: 3085-3094.

8. Choi DW. Calcium and excitotoxic neuronal injury. Ann N Y Acad Sci 1994; 747: 162-171.

9. Chomczyński P, Sacchi N. Single-step method of RNA isolation by acid guanidinium thiocyanate-phenol-chloroform extraction. Anal Biochem 1987; 162: 156-159.

10. Danbolt N. Glutamate uptake. Prog Neurobiol 2001; 65: 1-105.

11. Deng W, Wang H, Rosenberg PA, Volpe JJ, Jensen FE. Role of metabotropic glutamate receptors in oligodendrocyte excito- 
toxicity and oxidative stress. Proc Natl Acad Sci U S A 2004; 101: 7751-7756.

12. Geurts JJ, Wolswijk G, Bö L, van der Valk P, Polman CH, Troost D, Aronica E. Altered expression patterns of group I and II metabotropic glutamate receptors in multiple sclerosis. Brain 2003; 126: 1755-1766.

13. Groom AJ, Smith T, Turski L. Multiple sclerosis and glutamate. Ann N Y Acad Sci 2003; 993: 229-275.

14. Hartline DK. What is myelin? Neuron Glia Biol 2008; 4: 153-163.

15. Kerschensteiner M, Stadelmann C, Buddeberg BS, Merkler D, Bareyre FM, Anthony DC, Linington C, Brück W, Schwab ME. Targeting experimental autoimmune encephalomyelitis lesions to a predetermined axonal tract system allows for refined behavioral testing in an animal model of multiple sclerosis. Am J Pathol 2004; 164: 1455-1469.

16. Kursula P. Structural properties of proteins specific to the myelin sheath. Amino Acids 2008; 34: 175-185.

17. Lassmann H. Mechanisms of demyelination and tissue destruction in multiple sclerosis. Clin Neurol Neurosurg 2002; 104: 168 171.

18. Lassmann H. Mechanisms of white matter damage in multiple sclerosis. Glia 2014; 62: 1816-1830.

19. Ljubisavljevic S, Stojanovic I, Pavlovic D, Milojkovic M, Sokolovic D, Stevanovic I, Petrovic A. Suppression of the lipid peroxidation process in the CNS reduces neurological expression of experimentally induced autoimmune encephalomyelitis. Folia Neuropathol 2013; 51: 51-57.

20. Lucchinetti CF, Popescu BF, Bunyan RF, Moll NM, Roemer SF, Lassmann H, Brück W, Parisi JE, Scheithauer BW, Giannini C, Weigand SD, Mandrekar J, Ransohoff RM. Inflammatory cortical demyelination in early multiple sclerosis. N Engl J Med 2011; 365: 2188-2197.

21. Makarewicz D, Sulejczak D, Duszczyk M, Małek M, Stomka M, Lazarewicz JW. Delayed preconditioning with NMDA receptor antagonists in a rat model of perinatal asphyxia. Folia Neuropathol 2014; 52: 270-284.

22. Massella A, D'Intino G, Fernández M, Sivilia S, Lorenzini L, Giatti S, Melcangi RC, Calzà L, Giardino L. Gender effect on neurodegeneration and myelin markers in an animal model for multiple sclerosis. BMC Neurosci 2012; 13: 12.

23. Matute C, Alberdi E, Domercq M, Sánchez-Gómez MV, PérezSamartín A, Rodríguez-Antigüedad A, Pérez-Cerdá F. Excitotoxic damage to white matter. J Anat 2007; 210: 693-702.

24. Matute C, Domercq M, Fogarty DJ, Pascual de Zulueta M, Sánchez-Gómez MV. On how altered glutamate homeostasis may contribute to demyelinating diseases of the CNS. Adv Exp Med Biol 1999; 468: 97-107.

25. Morell P, Quarles RH. Myelin Formation, Structure and Biochemistry. In: Basic Neurochemistry: Molecular, Cellular and Medical Aspects. Siegel GJ, Agranoff BW, Albers RW, Fisher SK, Uhler MD (eds.). Lippincott-Raven, Philadelphia 1999; pp. 69-94.

26. Ohgoh M, Hanada T, Smith T, Hashimoto T, Ueno M, Yamanishi Y, Watanabe M., Nishizawa Y. Altered expression of glutamate transporters in experimental autoimmune encephalomyelitis. J Neuroimmunol 2002; 125: 170-178.

27. Otis TS, Brasnjo G, Dzubay JA, Pratap M. Interactions between glutamate transporters and metabotropic glutamate receptors at excitatory synapses in the cerebellar cortex. Neurochem Int 2004; 45: 537-544.

28. Paul C, Bolton C. Modulation of blood-brain-barrier dysfunction and neurological deficits during acute experimental allergic encephalomyelitis by the N-methyl-D-aspartate receptor antagonist memantine. J Pharmacol Exp Ther 2002; 302: 50-57.

29. Pitt D, Werner P, Raine CS. Glutamate excitotoxicity in a model of multiple sclerosis. Nat Med 2000; 6: 67-70.

30. Reindl M, Di Pauli F, Rostásy K, Berger T. The spectrum of MOG autoantibody-associated demyelinating diseases Nat Rev Neurol 2013; 9: 455-461.

31. Sospedra M, Martin R. Immunology of multiple sclerosis Annu Rev Immunol 2005; 23: 683-747.

32. Stys PK, Lipton SA. White matter NMDA receptors: an expected new therapeutic target? Trends Pharmacol Sci 2007; 28: 561566.

33. Sulkowski G, Dąbrowska-Bouta B, Chalimoniuk M, Strużyńska L. Effects of antagonists of glutamate receptors on pro-inflammatory cytokines in the brain cortex of rats subjected to experimental autoimmune encephalomyelitis. J Neuroimmunol 2013; 261: 67-76.

34. Sulkowski G, Dąbrowska-Bouta B, Strużyńska L. Modulation of neurological deficits and expression of glutamate receptors during experimental autoimmune encephalomyelitis after treatment with selected antagonists of glutamate receptors. Biomed Res Int 2013; article 186068.

35. Sulkowski G, Dąbrowska-Bouta B, Salińska E, Strużyńska L. Modulation of glutamate transport and receptor binding by glutamate receptor antagonists in EAE rat brain. PLoS One 2014; 9: article e113954.

36. Tronci E, Fidalgo C, Zianni E, Collu M, Stancampiano R, Morelli M, Gardoni F, Carta M. Effect of memantine on L-DOPA-induced dyskinesia in the 6-OHDA-lesioned rat model of Parkinson's disease. Neuroscience 2014; 265: 245-252.

37. Volbracht C, van Beek J, Zhu C, Blomgren K, Leist M. Neuroprotective properties of memantine in different in vitro and in vivo models of excitotoxicity. Eur J Neurosci 2006; 23: 2611-2622.

38. Werner P, Pitt D, Raine CS. Multiple sclerosis: altered glutamate homeostasis in lesions correlates with oligodendrocyte and axonal damage. Ann Neurol 2001; 50: 169-180. 